\title{
Perfusion by Arterial Spin labelling following Single dose Tadalafil In Small vessel disease (PASTIS): study protocol for a randomised controlled trial
}

Mathilde M. H. Pauls 1,2,3, Natasha Clarke ${ }^{1,4}$, Sarah Trippier ${ }^{4}$, Shai Betteridge ${ }^{5}$, Franklyn A. Howe', Usman Khan ${ }^{3}$, Christina Kruuse ${ }^{7}$, Jeremy B. Madigan ${ }^{1,6}$, Barry Moynihan ${ }^{8}$, Anthony C. Pereira ${ }^{3}$, Debbie Rolfe ${ }^{9}$, Egill Rostrup ${ }^{10}$, Caroline E. Haig ${ }^{11}$, Thomas R. Barrick', Jeremy D. Isaacs ${ }^{1,2,3}$ and Atticus H. Hainsworth ${ }^{1,2,3,12^{*}}$

\begin{abstract}
Background: Cerebral small vessel disease is a common cause of vascular cognitive impairment in older people, with no licensed treatment. Cerebral blood flow is reduced in small vessel disease. Tadalafil is a widely prescribed phosphodiesterase- 5 inhibitor that increases blood flow in other vascular territories. The aim of this trial is to test the hypothesis that tadalafil increases cerebral blood flow in older people with small vessel disease.

Methods/design: Perfusion by Arterial Spin labelling following Single dose Tadalafil In Small vessel disease (PASTIS) is a phase II randomised double-blind crossover trial. In two visits, 7-30 days apart, participants undergo arterial spin labelling to measure cerebral blood flow and a battery of cognitive tests, pre- and post-dosing with oral tadalafil (20 mg) or placebo. Sample size: 54 participants are required to detect a 15\% increase in cerebral blood flow in subcortical white matter ( $p<0.05,90 \%$ power). Primary outcomes are cerebral blood flow in subcortical white matter and deep grey nuclei. Secondary outcomes are cortical grey matter cerebral blood flow and performance on cognitive tests (reaction time, information processing speed, digit span forwards and backwards, semantic fluency).

Discussion: Recruitment started on 4th September 2015 and 36 participants have completed to date (19th April 2017). No serious adverse events have occurred. All participants have been recruited from one centre, St George's University Hospitals NHS Foundation Trust.
\end{abstract}

Trial registration: European Union Clinical Trials Register: EudraCT number 2015-001235-20. Registered on 13 May 2015.

Keywords: Tadalafil, Cerebral blood flow, Vascular cognitive impairment, Vascular dementia, Phosphodiesterase, MRl, Arterial spin labelling

\section{Background}

Cerebral small vessel disease (SVD) is a frequent cause of vascular cognitive impairment (VCI) in older adults [1-4]. There is currently no licensed treatment for SVD or for

\footnotetext{
* Correspondence: ahainsworth@sgul.ac.uk

${ }^{1}$ Neurosciences Research Centre, Molecular and Clinical Sciences Research Institute, St George's University of London, Cranmer Terrace, London SW17 ORE, UK

${ }^{2}$ Cell Biology and Genetics Research Centre, Molecular and Clinical Sciences Research Institute, St George's University of London, Cranmer Terrace, London SW17 ORE, UK

Full list of author information is available at the end of the article
}

VCI $[1,2]$. There is evidence derived from some previous studies to suggest that cerebral blood flow (CBF) is reduced in SVD, particularly in subcortical white matter [5-10]. We hypothesised that increasing CBF has the potential to be both a symptomatic and a disease-modifying treatment for SVD and VCI.

Phosphodiesterase type 5 inhibitors (PDE5i) such as sildenafil and tadalafil are well-established pharmacological vasodilators that cause enhanced nitric oxide-cyclic guanosine monophosphate signalling in peripheral small arteries [11-13]. PDE5i are widely used in treatment of 
erectile dysfunction and pulmonary hypertension [13]. PDE5 messenger RNA and protein are also found in human brain tissue [12, 14, 15]. Side-effect profiles of PDE5i are well-known, and the drugs are well-tolerated in the target population [16-18]. In a meta-analysis of 28 placebo-controlled trials [18], the overall incidence of myocardial infarction, cardiovascular death or cerebrovascular death in tadalafil-treated patients did not differ from placebo. The incidence of these adverse events was independent of dosing regimen and duration of tadalafil therapy (up to 27 months) [18]. The choice of tadalafil (over other PDE5i) was based on long plasma half-life (17 $\mathrm{h}$ in healthy adults) $[16,17]$ and established brain penetration (brain-to-plasma ratio 1:10 in rodents and primates) $[12,19]$. In this study, we will test whether single-dose tadalafil increases CBF in older people with neuroradiological and clinical evidence of SVD.

\section{Methods/design Objectives}

The aim of this study is to test the hypothesis that tadalafil increases cerebral blood flow in subcortical areas in older people with symptomatic SVD.

\section{Study design}

Perfusion by Arterial Spin labelling following Single dose Tadalafil In Small vessel disease (PASTIS) is a phase II double-blind crossover trial. Participants are randomised to order of treatment (tadalafil $20 \mathrm{mg}$, placebo; oral administration). Two visits are performed 7-30 days apart, with perfusion magnetic resonance imaging (MRI) and a battery of cognitive tests performed before and 3-5 h after dosing (see Fig. 1).

A Standard Protocol Items: Recommendations for Interventional Trials (SPIRIT) checklist is shown in Fig. 2 (see also Additional file 1).

\section{Trial endpoints}

The primary endpoints are change in regional CBF in two sub-cortical brain areas (deep white matter and deep grey nuclei). The secondary endpoints are (1) change in regional $\mathrm{CBF}$ in cortical grey matter, (2) change in neuropsychological test performance and (3) plasma tadalafil concentration dependence of any changes observed.

\section{Study setting}

Participants are recruited from St George's University Hospital NHS Foundation Trust and local Participant Identification Centre sites. All patient visits, data management and trial coordination are performed at St George's. PASTIS has been adopted into the UK National Institute for Health Research Clinical Research Network Portfolio.

\section{Screening Visit}

- Eligibility check

- Informed consent

- Clinical assessment

- $\quad$ MOCA, TOPF, NIHSS, MRS

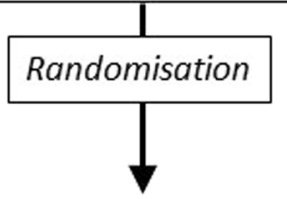

Visit $1>6$ months post stroke

- MRI \& Cognitive test battery pre-dose

- Dosing (placebo / tadalafil 20mg)

- MRI \& Cognitive test battery post-dose

Visit 2 7-30 days later

- MRI \& Cognitive test battery pre-dose

- Dosing (placebo / tadalafil 20mg)

- MRI \& Cognitive test battery post-dose

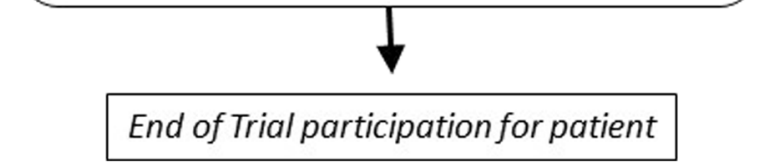

Fig. 1 MOCA Montreal Cognitive Assessment, TOPF Test of Premorbid Functioning, NIHSS National Institutes of Health Stroke Scale, MRS Modified Rankin Scale, MRI Magnetic resonance imaging

\section{Participant characteristics}

Participants are older people (men and women) without a diagnosis of dementia who have radiological and clinical evidence of symptomatic SVD. After informed consent is obtained, the following activities will occur at a screening visit (see Fig. 1):

1. Trial eligibility criteria check

2. Medical history

3. Concomitant medication checklist: medications, doses and frequencies

4. MRI suitability/contraindication checklist

5. Participant demographics, including ethnic origin

6. Next-of-kin and general practitioner contact details to be recorded if not already in medical notes, or check if still current and up-to-date

7. Affix Clinical Trials Alert sticker to front of the medical notes 


\begin{tabular}{|c|c|c|c|c|c|c|c|}
\hline \multirow[b]{2}{*}{ Study Procedures } & \multirow{2}{*}{$\begin{array}{l}\text { Visit } 0 \\
\text { Screening }\end{array}$} & \multicolumn{3}{|c|}{$\begin{array}{c}\text { Visit } 1 \text { - Day } 1(<60 \text { day } \\
\text { window })\end{array}$} & \multicolumn{3}{|c|}{ Visit 2 ( 7 - 30 days later) } \\
\hline & & $\begin{array}{l}\text { before } \\
\text { IMP } \\
\text { dose }\end{array}$ & $\begin{array}{l}\text { IMP } \\
\text { dosing }\end{array}$ & $\begin{array}{c}3-6 \mathrm{hrs} \\
\text { post } \\
\text { IMP } \\
\text { dosing }\end{array}$ & $\begin{array}{l}\text { before } \\
\text { IMP } \\
\text { dose }\end{array}$ & $\begin{array}{l}\text { IMP } \\
\text { dosing }\end{array}$ & $\begin{array}{c}\text { 3-6 } \\
\text { hours } \\
\text { post } \\
\text { IMP } \\
\text { dosing }\end{array}$ \\
\hline Informed consent & $x$ & & & & & & \\
\hline $\begin{array}{l}\text { Inclusion/exclusion } \\
\text { criteria }\end{array}$ & $\mathrm{x}$ & & & & & & \\
\hline Medical history & $\mathrm{x}$ & & & & & & \\
\hline Demographics & $\mathrm{x}$ & & & & & & \\
\hline Screening & $\mathrm{x}$ & & & & & & \\
\hline Modified Rankin Score & $\mathrm{x}$ & & & & & & \\
\hline MRI & & $\mathrm{x}$ & & $\mathrm{x}$ & $\mathrm{x}$ & & $\mathrm{x}$ \\
\hline $\begin{array}{l}\text { Neuropsychological Test } \\
\text { Batteries see Appendix } 3\end{array}$ & $\mathrm{x}$ & $\mathrm{x}$ & & $\mathrm{x}$ & $\mathrm{x}$ & & $\mathrm{x}$ \\
\hline $\begin{array}{l}\text { Dispensing/Administrati } \\
\text { on of IMP }\end{array}$ & & & $\mathrm{x}$ & & & $\mathrm{x}$ & \\
\hline Concomitant Medication & $\mathrm{x}$ & $\mathrm{x}$ & & & $\mathrm{x}$ & & \\
\hline Measure blood pressure & $\mathrm{x}$ & $x$ & & $\mathrm{x}$ & $\mathrm{x}$ & & $\mathrm{x}$ \\
\hline $\mathrm{FBC}^{*}$ & & $x$ & & $\mathrm{x}$ & $\mathrm{x}$ & & $\mathrm{x}$ \\
\hline $\begin{array}{l}\text { plasma samples for } \\
\text { Tadalafil drug levels }\end{array}$ & & & & $\mathrm{x}$ & & & $\mathrm{x}$ \\
\hline
\end{tabular}

Fig. 2 Standard Protocol Items: Recommendations for Interventional Trials (SPIRIT) checklist: schedule of enrolment, interventions and assessments in the Perfusion by Arterial Spin labelling following Single dose Tadalafil In Small vessel disease (PASTIS) trial. From PASTIS protocol version 4, 27 Jan 2016

8. Complete case report form screening page, ensuring the participant's trial identifier is included

9. Test of Premorbid Functioning (TOPF) to establish estimated levels of cognitive functioning pre-illness

10.National Institutes of Health Stroke Scale (NIHSS)

11.Montreal Cognitive Assessment (MoCA) to establish estimated levels of cognitive functioning

12.Record the modified Rankin Scale score (mRS)

\section{Inclusion criteria}

1. Radiological evidence of cerebral SVD, defined as MRI evidence of lacunar infarcts $(\leq 1.5 \mathrm{~cm}$ maximum diameter) and/or confluent deep white matter hyperintensities (WMH) ( $\geq$ grade 2 on Fazekas scale)

2. Clinical evidence of SVD, including the following:

a. Lacunar stroke syndrome with symptoms lasting $>24 \mathrm{~h}$, occurring $\geq 6$ months prior to visit 1 ; or

b. Transient ischaemic attack (TIA) lasting $<24 \mathrm{~h}$ with limb weakness, hemi-sensory loss or dysarthria $\geq 6$ months previously and with MRI diffusion-weighted imaging performed acutely showing lacunar infarction, or, if MRI is not performed within 10 days of TIA, lacunar infarct in an anatomically appropriate area

3. Age $\geq 50$ years

4. Imaging of the carotid arteries with Doppler ultrasound, computed tomographic angiography or 
magnetic resonance angiography in the previous 12 months demonstrating $<70 \%$ stenosis in both internal carotid arteries or $<50 \%$ stenosis in both internal carotids if measured in previous 12-60 months

\section{Exclusion criteria}

1. Known diagnosis of dementia

2. Cortical infarct $(>1.5 \mathrm{~cm}$ maximum diameter)

3. Systolic blood pressure (BP) $<90 \mathrm{mmHg}$ and/or diastolic $\mathrm{BP}<50 \mathrm{mmHg}$

4. Creatinine clearance $<30 \mathrm{ml} /$ minute

5. Severe hepatic impairment

6. History of lactose intolerance

7. Concomitant use of PDE5i (e.g., sildenafil, tadalafil, vardenafil)

8. Receiving nicorandil or nitrates (e.g., isosorbide mononitrate, glyceryl trinitrate)

9. Weight $>130 \mathrm{~kg}$

10.Uncontrolled cardiac failure

11.Persistent or paroxysmal atrial fibrillation

12. History of gastric ulceration

13. History of 'sick sinus syndrome' or other supraventricular cardiac conduction conditions

14.Uncontrolled chronic obstructive pulmonary disease 15.Stroke or TIA within 6 months

16.MRI not tolerated or contraindicated

17.Known monogenic causes of stroke (e.g., cerebral autosomal dominant arteriopathy with subcortical infarcts and leukoencephalopathy)

18.Unable to provide informed consent

\section{Randomisation}

The randomisation list will be generated by Sharp Clinical Services (http://www.sharpservices.com/our-facilities/sharp-clinical-services-wales/; Crickhowell, UK) and will be done in blocks, as detailed in the client study information form kept in the sponsor site file. The participants will be acting as their own controls. Each participant will receive on two separate occasions a placebo dose and a tadalafil 20-mg immediate dose which appear identical in size, shape, weight and colour.

The patient pack numbers on the pharmacy shelf correlate directly with the next available pack number on the blinded randomisation list held in the pharmacy site file. Each patient pack contains two bottles, labelled as bottle $\mathrm{A}$ and bottle $\mathrm{B}$. The randomisation list will be confidential to the trial statistician and will be summarised as treatment arm A and B, and not by tadalafil and placebo.

\section{Measurement of regional cerebral blood flow}

Whole-brain perfusion will be determined by pseudocontinuous arterial spin labelling (ASL) [20] in a 3-T
MRI scanner (Achieva TX MRI scanner, Philips Medical Systems, Eindhoven, Netherlands). A total of 20-minute pseudo-continuous ASL acquisition time will be used to provide an adequate signal-to-noise ratio for CBF quantification in white matter. Other image data acquired will be as follows: an $\mathrm{M}_{0}$ image, to enable quantification of CBF; high-resolution 3D T1-weighted images for identification of grey and white matter regions of interest (including deep grey matter structures) for ASL analysis [20] and to map the ASL data to a standard brain atlas; fluid-attenuated inversion recovery (FLAIR) for delineation of WMH; and susceptibility-weighted imaging for detection of micro-haemorrhages. These will provide participant-specific WMH load and location of WMH. Total scanning time is under 60 minutes per MRI session.

\section{Cognitive testing}

Scores derived from the TOPF and MoCA instruments are recorded at the screening visit. These are included in the analyses as baseline data. They are not used as inclusion or exclusion criteria.

At the two dosing visits, the following neuropsychological tests are used: the Reaction Time subtest of the Cambridge Cognition Cambridge Neuropsychological Test Automated Battery, the Speed of Information Processing subtest of the Brain Injury Rehabilitation Trust Memory and Information Processing Battery, the Digit Span forwards and backwards subtest of the Repeatable Battery for the Assessment of Neuropsychological Status (RBANS), and the Semantic Fluency subtest of RBANS.

\section{Biochemical analyses}

A blood sample is taken at the end of visits 1 and 2 for haematocrit and full blood count analysis. Plasma samples are stored at $-80{ }^{\circ} \mathrm{C}$ for subsequent analysis of plasma tadalafil concentration.

\section{Details of the intervention}

Each participant pack contains one bottle containing a single tadalafil 20-mg capsule and one identical bottle containing a single matched placebo capsule. At each visit, participants undergo cognitive tests and the first MRI scanning session of the day. Participants are then observed to swallow the appropriate investigational medicinal product (IMP) capsule and receive a standard light lunch (450-750 kcal and $500 \mathrm{ml}$ of fluid). They undergo an equivalent, parallel version of the cognitive tests and the second MRI session of the day 3-5 h later. All participants are given a 24-h emergency contact card with the study title, details of the IMP, their participant trial number, investigator's contact details, and out-ofhours contact details (see Fig. 3). 
All those involved in the study (researchers, radiologists, pharmacists and participants) are blinded to treatment allocation for the duration of the study. Emergency un-blinding will take place in circumstances such as serious adverse events (SAEs). Any SAEs and safety endpoints will be reported in line with clinical trial regulations (SI2004/1031) and the sponsor's procedures. We do not anticipate any serious adverse reactions to the medication, because tadalafil is widely used clinically and is well-tolerated. The starting point for SAE monitoring is the first intervention visit, ending 5 days after the second visit (based on a drug elimination period of 6 half-lives for the study medication, using a 20 -h half-life for tadalafil).

\section{Power calculation}

On the basis of previous ASL studies of regional CBF, we estimate baseline perfusion of $30( \pm 10) \mathrm{ml} / 100 \mathrm{~g} / \mathrm{mi}$ nute $($ mean $\pm \mathrm{SD})$ in subcortical white matter and 70
$( \pm 15) \mathrm{ml} / 100 \mathrm{~g} / \mathrm{minute}$ in deep grey nuclei $[21,22]$. To detect a treatment effect of $15 \%$ (mean paired difference) with statistical power of $90 \%$, a sample size of 24 is required in deep grey matter nuclei and 54 in subcortical white matter. We aim to recruit a target cohort of 54 .

\section{Statistical analysis}

Baseline characteristics (age, sex, ethnic group, baseline BP, mRS score, NIHSS, TOPF, MoCA) will be summarised as mean (SD) or median (Q1, Q3) for continuous variables, depending on distribution, and as number (percent) for categorical variables. Changes in outcome variables will be calculated for each participant at each visit as post-dose value minus pre-dose value. Data will be analysed using a linear mixed effects regression model with fixed effects for treatment (drug vs. placebo), visit (visit 1, visit 2), treatment sequence and baseline response, as well as a random effect for participant nested within treatment sequence. Carry-over will be

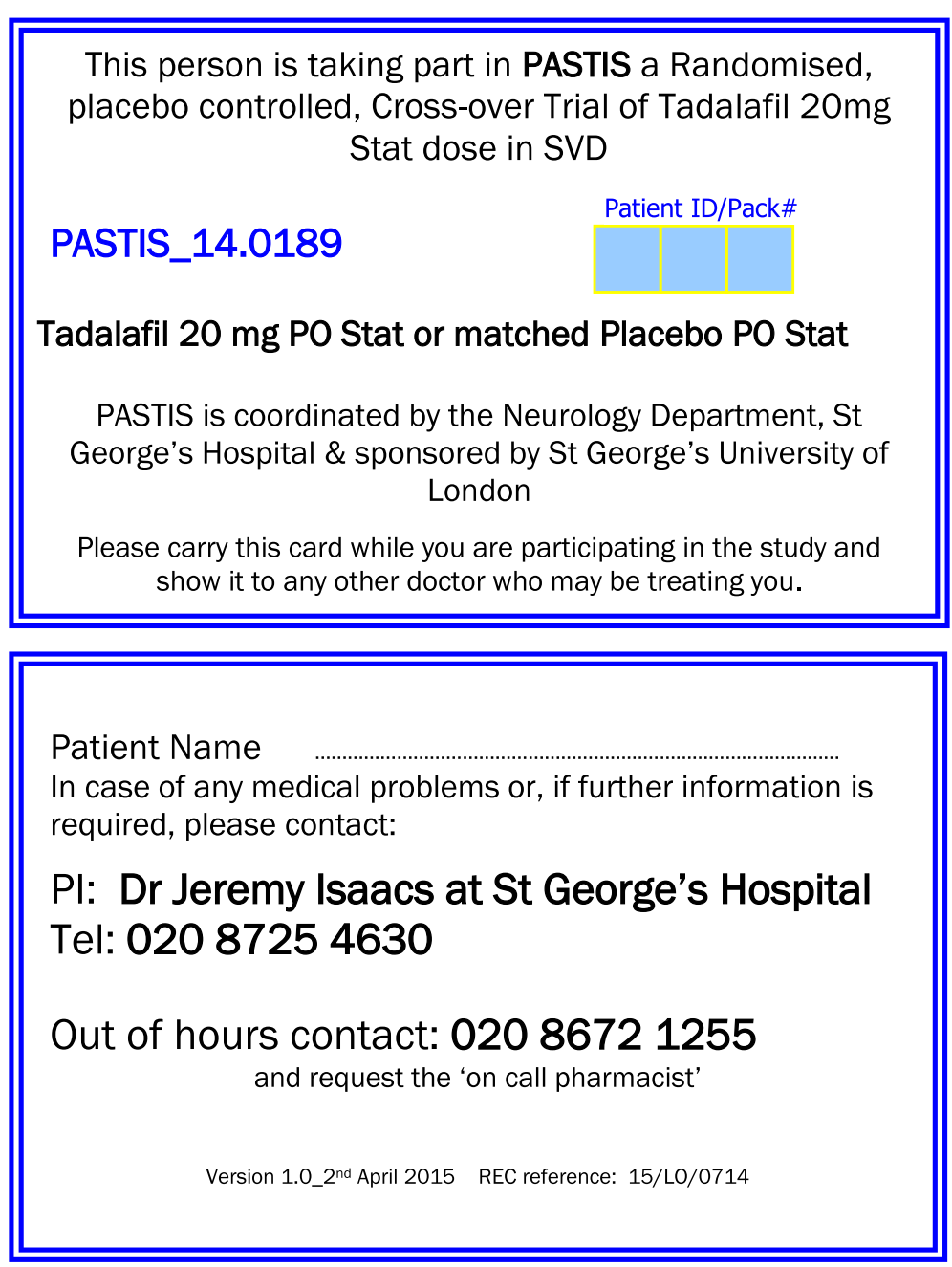

Fig. 3 PASTIS trial emergency 24-hour contact card, supplied to each participant 
investigated by the treatment-by-visit interaction. If statistically significant, data from each visit will be analysed separately within linear regression models adjusted for treatment and pre-dose value. Clinical variables and other possible confounders (e.g., BP at the time of the scan) will be included in the linear mixed effects models as adjustment variables. These will be pre-specified in the statistical analysis plan.

All analyses will be done on an intention-to-treat basis, and no adjustment will be made for missing data. Statistical analyses will be performed using $\mathrm{SAS}^{\bullet}$ for Windows version 9.3 or later software (SAS Institute, Cary, NC, USA). A $p$ value $>0.05$ indicates the absence of a statistically significant effect.

\section{Data monitoring}

Monitoring is performed by the sponsor clinical trials monitor in accordance with an agreed risk-based monitoring plan. Case report form entries are verified against the source documents and the participants' medical notes. All data are entered directly from case report forms to the PASTIS Microsoft Access (Microsoft Corp., Redmond, WA, USA) database by the PASTIS research team. Data transfer from the case report form will be double-checked, and where corrections are required, these will carry a full audit trail and justification. Trial data storage conforms to St George's institutional information governance policies. Trial data, evidence of monitoring and system audits will be made available for inspection by the sponsor and regulatory authorities as required.

\section{Discussion}

In this randomised, double-blind, crossover phase II study, we will test whether tadalafil $(20 \mathrm{mg})$ increases CBF in older people with SVD. Tadalafil was chosen over other PDE5i (e.g., sildenafil or vardenafil) because of the documented brain penetration [12, 19] and longer plasma half-life of tadalafil $[16,17]$. In the present trial, we are simply testing for acute changes in response to a single dose of tadalafil. For this purpose, a crossover design appeared optimal. In the event that a positive outcome is detected in the present study, it appears likely that a subsequent study testing tadalafil over a longer dosing period will be required. This will be needed to explore whether any tadalafil-mediated actions are maintained with chronic dosing and to test for any additional adverse reactions in participants who are likely to be taking concomitant stroke medications.

ASL was chosen to quantify regional CBF because it does not require injected radioisotopes or gadolinium compounds as tracers [20-22]. This MRI-based approach also enables acquisition of high-resolution 3D T1-weighted images, T2-weighted FLAIR images and susceptibility-weighted imaging. The neuropsychological tests that are used were chosen because each has four parallel versions of the test to be applied at each screening point (Fig. 1). The cognitive tests used measure processing speed, attention and executive function, which are affected in SVD, as well as working memory and semantic fluency. Nevertheless, it may be difficult to detect cognitive changes in such short-term follow-up as is employed in the present study. The cognitive data obtained from this trial may be of value in assessing sample size and feasibility for any subsequent trial of tadalafil in relation to cognitive function.

In addition to the European Union Clinical Trials Register (EudraCT number 2015-001235-20, date of registration 13 May 2015), the trial has been registered with ClinicalTrials.gov (NCT02450253, date of registration 18 May 2015). No SAEs have been observed so far. Inadvertent un-blinding due to the erectile effects of tadalafil has not occurred so far as we are aware. Spontaneous penile erection has been reported in a modest fraction (11\%) of subjects taking $20 \mathrm{mg}$ of tadalafil [16, 17]. PASTIS is the first phase II clinical trial of a selective PDE5i in older people with symptomatic SVD. Outcome data are expected in late 2017 and may inform a larger trial for re-purposing of tadalafil in SVD and VCI.

\section{Trial status}

The trial commenced on 4th September 2015. The PASTIS trial is ongoing. Patient recruitment has not been completed. As of 19 April 2017, 36 participants have completed the protocol.

\section{Additional file}

Additional file 1: SPIRIT 2013 checklist: recommended items to address in a clinical trial protocol and related documents. (DOC $120 \mathrm{~kb}$ )

\section{Abbreviations}

ASL: Arterial spin labelling; BP: Blood pressure; CBF: Cerebral blood flow; FLAIR: Fluid-attenuated inversion recovery; IMP: Investigational medicinal product; MoCA: Montreal Cognitive Assessment; MRI: Magnetic resonance imaging; mRS: Modified Rankin Scale; NIHSS: National Institutes of Health Stroke Scale; PASTIS: Perfusion by Arterial Spin labelling following Single dose Tadalafil In Small vessel disease; PDE5i: Phosphodiesterase type 5 inhibitor; RBANS: Repeatable Battery for the Assessment of Neuropsychological Status; SAE: Serious adverse event; SPIRIT: Standard Protocol Items: Recommendations for Interventional Trials; SVD: Small vessel disease; TIA: Transient ischaemic attack; TOPF: Test of premorbid functioning; VCl: Vascular cognitive impairment; WMH: White matter hyperintensities

\section{Acknowledgements}

We are very grateful to our colleagues at St George's Hospital and St George's University of London for their support of the PASTIS study.

\section{Funding}

The study is jointly funded by the Alzheimer's Drug Discovery Foundation and the Alzheimer's Society (grant reference 20140901). NC is funded by MRC doctoral training programme grant number MR/N013638/1. The funders have no input into trial design, data collection or data analysis. 


\section{Availability of data and materials}

Not applicable.

\section{Authors' contributions}

JDI, TRB, FAH, DR, SB, JBM, BM, UK, ACP, CK, ER and AHH contributed to study design. MMHP, ST and NC performed data collection. MMHP, TRB and CEH carried out data analysis. MMHP and NC drafted the manuscript. All authors contributed to revising the manuscript. AHH prepared the final manuscript. All authors read and approved the final manuscript.

\section{Authors' information}

MMHP is a clinical research fellow at St George's University Hospitals NHS Foundation Trust, London. NC is a neuroscience researcher at St George's University of London. ST is a stroke research clinical studies coordinator at St George's University Hospitals NHS Foundation Trust, London. SB is a consultant neuropsychologist at St George's University Hospitals NHS Foundation Trust, London. FAH is a professor of medical physics at St George's University of London. UK is a consultant neurologist at St George's University Hospitals NHS Foundation Trust, London. CK is a consultant neurologist at Herlev Gentofte Hospital and associate professor of stroke medicine at University of Copenhagen, Denmark. JBM is a consultant neuroradiologist at St George's University Hospitals NHS Foundation Trust, London. BM is a consultant is stroke medicine at Beaumont Hospital, Dublin. ACP is a consultant neurologist at St George's University Hospitals NHS Foundation Trust, London. DR is a regulatory assurance manager, St George's University of London. ER is a research consultant of nuclear medicine, Rigshospitalet Glostrup, Denmark. CEH is a biostatistician at the Robertson Centre for Biostatistics, University of Glasgow. TRB is a senior lecturer in image analysis at St George's University of London. JDI is a Consultant neurologist and is clinical principal investigator on the PASTIS trial. AHH is a reader in cerebrovascular disease at St George's University of London and is chief investigator on the PASTIS trial.

\section{Competing interests}

The authors declare that they have no competing interests.

\section{Consent for publication}

Not applicable.

\section{Ethics approval and consent to participate}

The PASTIS Study received NHS National Research Ethics Service approval on 6 May 2015 (London-Brent Research Ethics Committee reference 15/LO/ 0714). It received Medicines and Healthcare products Regulatory Agency authorisation (reference 16745/0222/001-0001) on 5 June 2015 and St George's University of London research and development approval (protocol number 14.0189) on 4 September 2015. Informed consent to participate is obtained from all participants in the study. The trial opened for recruitment on 4 September 2015, and the first participant was enrolled on 14 September 2015. The PASTIS trial is sponsored by the Joint Research and Enterprise Office, St George's University of London, Cranmer Terrace, London SW17 ORE, UK. A representative of the sponsor (DR) has had input into trial design and has contributed to this report.

\section{Publisher's Note}

Springer Nature remains neutral with regard to jurisdictional claims in published maps and institutional affiliations.

\section{Author details}

${ }^{1}$ Neurosciences Research Centre, Molecular and Clinical Sciences Research Institute, St George's University of London, Cranmer Terrace, London SW17 ORE, UK. ${ }^{2}$ Cell Biology and Genetics Research Centre, Molecular and Clinical Sciences Research Institute, St George's University of London, Cranmer Terrace, London SW17 ORE, UK. ${ }^{3}$ Department of Neurology, St George's University Hospitals NHS Foundation Trust, Blackshaw Road, London SW17 OQT, UK. ${ }^{4}$ Stroke Clinical Research Network, St George's University Hospitals NHS Foundation Trust, Blackshaw Road, London SW17 OQT, UK. ${ }^{5}$ Department of Neuropsychology, St George's University Hospitals NHS Foundation Trust, Blackshaw Road, London SW17 0QT, UK. 'ㄹepartment of Neuroradiology, St George's University Hospitals NHS Foundation Trust, Blackshaw Road, London SW17 OQT, UK. 'Department of Neurology, Herlev Hospital, Herlev Ringvej 75, 2730 Herlev, Denmark. ${ }^{8}$ Beaumont Hospital, Beaumont, Dublin 9,
Ireland. ${ }^{9}$ Joint Research and Enterprise Office, St George's University of London, Cranmer Terrace, London SW17 ORE, UK. ${ }^{10}$ Department of Clinical Physiology and Nuclear Medicine, Rigshospitalet, Nordre Ringvej 57, DK-2600 Glostrup, Denmark. ${ }^{11}$ Robertson Centre for Biostatistics, University of Glasgow, Glasgow G12 8QQ, UK. ${ }^{12}$ Cerebrovascular Disease, St George's University of London, Cranmer Terrace, London SW17 ORE, UK.

Received: 4 January 2017 Accepted: 4 May 2017

Published online: 22 May 2017

\section{References}

1. O'Brien JT, Thomas A. Vascular dementia. Lancet. 2015;386:1698-706.

2. Pantoni L. Cerebral small vessel disease: from pathogenesis and clinical characteristics to therapeutic challenges. Lancet Neurol. 2010;9:689-701.

3. Esiri MM, Wilcock GK, Morris JH. Neuropathological assessment of the lesions of significance in vascular dementia. J Neurol Neurosurg Psychiatry. 1997;63:749-53.

4. Ighodaro ET, Abner EL, Fardo DW, Lin AL, Katsumata Y, Schmitt FA, et al. Risk factors and global cognitive status related to brain arteriolosclerosis in elderly individuals. J Cereb Blood Flow Metab. 2017;37:201-16.

5. Markus HS, Lythgoe DJ, Ostegaard L, O'Sullivan M, Williams SC. Reduced cerebral blood flow in white matter in ischaemic leukoaraiosis demonstrated using quantitative exogenous contrast based perfusion MRI. J Neurol Neurosurg Psychiatry. 2000;69:48-53.

6. O'Sullivan M, Lythgoe DJ, Pereira AC, Summers PE, Jarosz JM, Williams SC, et al. Patterns of cerebral blood flow reduction in patients with ischemic leukoaraiosis. Neurology. 2002;59:321-6.

7. Schuff N, Matsumoto S, Kmiecik J, Studholme C, Du A, Ezekiel F, et al. Cerebral blood flow in ischemic vascular dementia and Alzheimer's disease, measured by arterial spin-labeling magnetic resonance imaging. Alzheimers Dement. 2009;5:454-62.

8. Yao H, Sadoshima S, Ibayashi S, Kuwabara Y, Ichiya Y, Fujishima M. Leukoaraiosis and dementia in hypertensive patients. Stroke. 1992;23:1673-7.

9. Bernbaum M, Menon BK, Fick G, Smith EE, Goyal M, Frayne R, et al. Reduced blood flow in normal white matter predicts development of leukoaraiosis. J Cereb Blood Flow Metab. 2015;35:1610-5.

10. Arba F, Mair G, Carpenter T, Sakka E, Sandercock PA, Lindley Rl, et al. Cerebral white matter hypoperfusion increases with small-vessel disease burden: data from the Third International Stroke Trial. J Stroke Cerebrovasc Dis. doi: 10.1016/j.jstrokecerebrovasdis.2017.03.002.

11. Conti M, Beavo J. Biochemistry and physiology of cyclic nucleotide phosphodiesterases: essential components in cyclic nucleotide signaling. Annu Rev Biochem. 2007;76:481-511.

12. Garcia-Osta A, Cuadrado-Tejedor M, Garcia-Barroso C, Oyarzabal J, Franco R. Phosphodiesterases as therapeutic targets for Alzheimer's disease. ACS Chem Neurosci. 2012;3:832-44.

13. Maurice DH, Ke H, Ahmad F, Wang Y, Chung J, Manganiello VC. Advances in targeting cyclic nucleotide phosphodiesterases. Nat Rev Drug Discov. 2014; 13:290-314.

14. Lakics V, Karran EH, Boess FG. Quantitative comparison of phosphodiesterase mRNA distribution in human brain and peripheral tissues. Neuropharmacology. 2010;59:367-74.

15. Kruuse C, Khurana TS, Rybalkin SD, Birk S, Engel U, Edvinsson L, et al. Phosphodiesterase 5 and effects of sildenafil on cerebral arteries of man and guinea pig. Eur J Pharmacol. 2005;521:105-14.

16. Forgue ST, Patterson BE, Bedding AW, Payne CD, Phillips DL, Wrishko RE, et al. Tadalafil pharmacokinetics in healthy subjects. Br I Clin Pharmacol. 2006; 61:280-8.

17. Forgue ST, Phillips DL, Bedding AW, Payne CD, Jewell H, Patterson BE, et al. Effects of gender, age, diabetes mellitus and renal and hepatic impairment on tadalafil pharmacokinetics. Br J Clin Pharmacol. 2007; 63:24-35.

18. Kloner RA, Jackson G, Hutter AM, Mittleman MA, Chan M, Warner MR, et al. Cardiovascular safety update of tadalafil: retrospective analysis of data from placebo-controlled and open-label clinical trials of tadalafil with as needed, three times-per-week or once-a-day dosing. Am J Cardiol. 2006;97:1778-84.

19. Garcia-Barroso C, Ricobaraza A, Pascual-Lucas M, Unceta N, Rico AJ, Goicolea $M A$, et al. Tadalafil crosses the blood-brain barrier and reverses cognitive dysfunction in a mouse model of AD. Neuropharmacology. 2013;64:114-23. 
20. Alsop DC, Detre JA, Golay X, Gunther M, Hendrikse J, Hernandez-Garcia L, et al. Recommended implementation of arterial spin-labeled perfusion MRI for clinical applications: a consensus of the ISMRM perfusion study group and the European consortium for ASL in dementia. Magn Reson Med. 2015;73: 102-16.

21. D'haeseleer M, Beelen R, Fierens Y, Cambron M, Vanbinst AM, Verborgh C, et al. Cerebral hypoperfusion in multiple sclerosis is reversible and mediated by endothelin-1. Proc Natl Acad Sci U S A. 2013;110:5654-8.

22. Colloby SJ, Firbank MJ, He J, Thomas AJ, Vasudev A, Parry SW, et al. Regional cerebral blood flow in late-life depression: arterial spin labelling magnetic resonance study. Br J Psychiatry. 2012;200:150-5.

Submit your next manuscript to BioMed Central and we will help you at every step:

- We accept pre-submission inquiries

- Our selector tool helps you to find the most relevant journal

- We provide round the clock customer support

- Convenient online submission

- Thorough peer review

- Inclusion in PubMed and all major indexing services

- Maximum visibility for your research

Submit your manuscript at www.biomedcentral.com/submit
Biomed Central 